\title{
Characteristic integrals in 3D and linear degeneracy
}

E.V. Ferapontov, J. Moss

To cite this article: E.V. Ferapontov, J. Moss (2014) Characteristic integrals in 3D and linear degeneracy, Journal of Nonlinear Mathematical Physics 21:2, 214-224, DOI: https://doi.org/10.1080/14029251.2014.900993

To link to this article: https://doi.org/10.1080/14029251.2014.900993

Published online: 04 January 2021 


\title{
Characteristic integrals in 3D and linear degeneracy
}

\author{
E.V. Ferapontov and J. Moss \\ Department of Mathematical Sciences, Loughborough University \\ Loughborough, Leicestershire LE11 3TU, UK \\ E.V.Ferapontov@lboro.ac.uk,J.Moss@lboro.ac.uk
}

Received 19 December 2013

Accepted 22 January 2014

\begin{abstract}
Conservation laws vanishing along characteristic directions of a given system of PDEs are known as characteristic conservation laws, or characteristic integrals. In 2D, they play an important role in the theory of Darboux-integrable equations. In this paper we discuss characteristic integrals in 3D and demonstrate that, for a class of second order linearly degenerate dispersionless integrable PDEs, the corresponding characteristic integrals are parametrised by points on the Veronese variety.
\end{abstract}

Keywords: Characteristic integrals, principal symbol, linear degeneracy, dispersionless integrability, Veronese variety

2000 Mathematics Subject Classification: 35A30, 37K10

\section{Introduction}

Let $\Sigma$ be a partial differential equation (PDE) in $n$ independent variables $x_{1}, \ldots, x_{n}$. A conservation law is an $(n-1)$-form $\Omega$ which is closed on the solutions of $\Sigma$ : $d \Omega=0 \bmod \Sigma$. Since any $(n-1)$ form in $n$ variables possesses a unique annihilating direction, there exists a vector field $F$ such that $\Omega(F)=0$. We say that $\Omega$ is a characteristic integral (conservation law) if $F$ is a characteristic direction of $\Sigma$. ${ }^{\text {a }}$ If a conservation law is represented in conventional form,

$$
\left(F_{1}\right)_{x_{1}}+\cdots+\left(F_{n}\right)_{x_{n}}=0 \bmod \Sigma,
$$

the corresponding vector field is $F=\left(F_{1}, \ldots, F_{n}\right)$. The characteristic condition becomes particularly simple for scalar second order PDEs, in which case $F$ can be interpreted as a null vector of the conformal structure defined by the principal symbol of the equation.

In two dimensions, characteristic integrals arise in the context of Darboux integrability, see e.g. $[27,31]$ and references therein. Having in mind a generalisation of Darboux integrability to higher dimensions (see $[2,3,12,13,23]$ for some steps in this direction), in this paper we develop the concept of characteristic integrals in 3D. We believe it to be of interest in its own.

Let us begin with illustrating examples.

Example 1.1. Consider the $2+1$ dimensional wave equation,

$$
u_{t t}=u_{x x}+u_{y y} .
$$

It possesses four first order conservation laws,

$$
\left(u_{x}\right)_{x}+\left(u_{y}\right)_{y}-\left(u_{t}\right)_{t}=0
$$

${ }^{a}$ The set of characteristic directions is projectively dual to the more conventional variety of characteristic covectors determined by the principal symbol of the equation. 


$$
\begin{aligned}
& \left(u_{x}^{2}+u_{t}^{2}-u_{y}^{2}\right)_{x}+\left(2 u_{x} u_{y}\right)_{y}-\left(2 u_{x} u_{t}\right)_{t}=0, \\
& \left(2 u_{y} u_{x}\right)_{x}-\left(u_{x}^{2}-u_{y}^{2}-u_{t}^{2}\right)_{y}-\left(2 u_{y} u_{t}\right)_{t}=0, \\
& \left(2 u_{t} u_{x}\right)_{x}+\left(2 u_{t} u_{y}\right)_{y}-\left(u_{x}^{2}+u_{y}^{2}+u_{t}^{2}\right)_{t}=0 .
\end{aligned}
$$

Let us denote them

$$
\left(f_{i}\right)_{x}+\left(g_{i}\right)_{y}+\left(h_{i}\right)_{t}=0,
$$

$i=1, \ldots, 4$. Taking their linear combination with constant coefficients $J_{1}, \ldots, J_{4}$, and adding trivial conservation laws, we obtain the expression $\left(F_{1}\right)_{x}+\left(F_{2}\right)_{y}+\left(F_{3}\right)_{t}=0$ where

$$
F_{1}=J_{i} f_{i}-J_{5} u_{y}+J_{6} u_{t}+J_{8}, \quad F_{2}=J_{i} g_{i}+J_{5} u_{x}-J_{7} u_{t}+J_{9}, \quad F_{3}=J_{i} h_{i}-J_{6} u_{x}+J_{7} u_{y}+J_{10},
$$

(summation over $i=1, \ldots, 4$ is assumed). Here the constants $J_{5}, J_{6}, J_{7}$ correspond to trivial conservation laws of the form $\left(u_{x}\right)_{y}-\left(u_{y}\right)_{x}=0$, etc., and $J_{8}, J_{9}, J_{10}$ are three extra arbitrary constants. Although the constants $J_{5}-J_{10}$ correspond to trivial conservation laws, they effect non-trivially the characteristic condition, $F g^{-1} F^{t}=0$, where $g$ is the $3 \times 3$ symmetric matrix of the corresponding principal symbol,

$$
g=\left(\begin{array}{ccc}
-1 & 0 & 0 \\
0 & -1 & 0 \\
0 & 0 & 1
\end{array}\right),
$$

(in this particular example $g$ coincides with $g^{-1}$ ). The characteristic condition imposes a system of quadratic constraints for $J_{1}, \ldots, J_{10}$, which specify a Veronese threefold $V^{3} \subset \mathbb{P}^{9}$ with parametric equations

$$
\begin{gathered}
J_{1}=\frac{\sqrt{2}}{4}(\gamma \alpha+\gamma \beta+\delta \alpha-\delta \beta), \quad J_{2}=-\alpha \beta, \quad J_{3}=\frac{\alpha^{2}-\beta^{2}}{2}, \quad J_{4}=-\frac{\alpha^{2}+\beta^{2}}{2}, \\
J_{5}=\frac{\sqrt{2}}{2}(\gamma \alpha-\gamma \beta-\delta \alpha-\delta \beta), \quad J_{6}=-\frac{\sqrt{2}}{2}(\gamma \alpha+\gamma \beta-\delta \alpha+\delta \beta) \\
J_{7}=\frac{\sqrt{2}}{2}(\gamma \alpha-\gamma \beta+\delta \alpha+\delta \beta), \quad J_{8}=\frac{\delta^{2}-\gamma^{2}}{2}, \quad J_{9}=\delta \gamma, \quad J_{10}=\frac{\gamma^{2}+\delta^{2}}{2}
\end{gathered}
$$

We use $\alpha, \beta, \gamma, \delta$ for homogeneous coordinates in $\mathbb{P}^{3}$, and $J_{1}, \ldots, J_{10}$ for homogeneous coordinates in $\mathbb{P}^{9}$. Recall that the Veronese threefold $V^{3}$ is the image of the projective imbedding of $\mathbb{P}^{3}$ into $\mathbb{P}^{9}$ defined by a complete system of quadrics. Thus, we have a whole $V^{3}$-worth of characteristic integrals. It turns out that this example is not isolated, and similar phenomena take place for other classes of 3D linearly degenerate dispersionless integrable PDEs.

Example 1.2. Let us consider the equation

$$
\mu u_{t} u_{x y}+v u_{y} u_{x t}+\eta u_{x} u_{y t}=0
$$

$\mu+v+\eta=0$, which appeared in the context of Veronese webs in 3D [29], as well as in the theory of Einstein-Weyl geometries of hyper-CR type [6]. This equation possesses four first order 
conservation laws,

$$
\begin{gathered}
\eta\left(u_{y} u_{t}\right)_{x}+v\left(u_{x} u_{t}\right)_{y}+\mu\left(u_{x} u_{y}\right)_{t}=0, \\
v\left(\frac{u_{y}}{u_{t}}\right)_{x}+\eta\left(\frac{u_{x}}{u_{t}}\right)_{y}=0 \\
\mu\left(\frac{u_{t}}{u_{y}}\right)_{x}+\eta\left(\frac{u_{x}}{u_{y}}\right)_{t}=0 \\
\mu\left(\frac{u_{t}}{u_{x}}\right)_{y}+v\left(\frac{u_{y}}{u_{x}}\right)_{t}=0 .
\end{gathered}
$$

Let us denote them

$$
\left(f_{i}\right)_{x}+\left(g_{i}\right)_{y}+\left(h_{i}\right)_{t}=0,
$$

$i=1, \ldots, 4$. Taking their linear combination with coefficients $J_{1}, \ldots, J_{4}$, and adding trivial conservation laws, we obtain the expression $\left(F_{1}\right)_{x}+\left(F_{2}\right)_{y}+\left(F_{3}\right)_{t}=0$ where

$$
F_{1}=J_{i} f_{i}-J_{5} u_{y}+J_{6} u_{t}+J_{8}, \quad F_{2}=J_{i} g_{i}+J_{5} u_{x}-J_{7} u_{t}+J_{9}, \quad F_{3}=J_{i} h_{i}-J_{6} u_{x}+J_{7} u_{y}+J_{10} .
$$

As in Example 1.1, the constants $J_{5}-J_{10}$ correspond to trivial conservation laws. The characteristic condition takes the form $\mathrm{Fg}^{-1} \mathrm{~F}^{t}=0$ where $g$ is the $3 \times 3$ symmetric matrix of the corresponding principal symbol:

$$
g=\left(\begin{array}{ccc}
0 & \mu u_{t} & v u_{y} \\
\mu u_{t} & 0 & \eta u_{x} \\
v u_{y} & \eta u_{x} & 0
\end{array}\right)
$$

The characteristic condition imposes a system of quadratic constraints for $J_{1}, \ldots, J_{10}$, which specify a Veronese threefold $V^{3} \subset \mathbb{P}^{9}$ with parametric equations

$$
\begin{gathered}
J_{1}=\alpha^{2}, \quad J_{2}=\frac{1}{4 v \eta} \beta^{2}, \quad J_{3}=\frac{1}{4 \eta \mu} \delta^{2}, \quad J_{4}=\frac{1}{4 v \mu} \gamma^{2}, \\
J_{5}=\alpha \beta, \quad J_{6}=\alpha \delta, \quad J_{7}=\alpha \gamma, \quad J_{8}=-\frac{1}{2 \eta} \beta \delta, \quad J_{9}=-\frac{1}{2 v} \beta \gamma, \quad J_{10}=-\frac{1}{2 \mu} \delta \gamma .
\end{gathered}
$$

Further examples of this type are given in the Appendix.

The structure of the paper is as follows:

In Sect. 2 we briefly review the case of 2D hydrodynamic type systems, where the existence of characteristic integrals is known to imply linear degeneracy.

In Sect. 3 we consider characteristic integrals of second order quasilinear PDEs in 3D. We prove that the requirement of the existence of 'sufficiently many' integrals of this type implies linear degeneracy. For all linearly degenerate integrable cases, we obtain $V^{3}$-worth of characteristic integrals.

We hope that the results of this paper may be useful for the theory of multi-dimensional Darboux-integrable equations (yet to be constructed). 


\section{Characteristic integrals in 2D and linear degeneracy}

For definiteness we restrict the discussion to systems of hydrodynamic type,

$$
u_{t}^{i}=v_{j}^{i}(\mathbf{u}) u_{x}^{i},
$$

where $\mathbf{u}=\left(u^{1}, \ldots, u^{n}\right)$ denotes dependent variables, and $v=v_{j}^{i}$ is an $n \times n$ matrix. Let $\lambda^{i}$ be the eigenvalues (characteristic speeds) of $v$, and let $\xi^{i}$ be the corresponding eigenvectors, so that $v \xi^{i}=$ $\lambda^{i} \xi^{i}$. Characteristic directions are defined as $d x+\lambda^{i} d t=0$, and the characteristic integral in $i$-th direction is a 1-form $h(\mathbf{u})\left(d x+\lambda^{i} d t\right)$ which is closed on solutions of (2.1). We will assume that the density $h$ depends on $\mathbf{u}$ only, although, in principle, nontrivial dependence on higher-order $x$ derivatives of $\mathbf{u}$ may also be allowed. Recall that the $i$-th characteristic direction is called linearly degenerate if the Lie derivative of $\lambda^{i}$ in the direction of the corresponding eigenvector $\xi^{i}$ vanishes, $L_{\xi^{i}} \lambda^{i}=0$. The following result is well-known:

Proposition 2.1. If there exists a characteristic integral in the $i$-th direction, then the corresponding characteristic speed $\lambda^{i}$ must be linearly degenerate.

Proof. The closedness of $h(\mathbf{u})\left(d x+\lambda^{i} d t\right)$ is equivalent to $h_{t}=\left(\lambda^{i} h\right)_{x}$. This implies

$$
(\nabla h) v=h \nabla \lambda^{i}+\lambda^{i} \nabla h
$$

where $\nabla=\left(\partial_{u^{1}}, \ldots, \partial_{u^{n}}\right)$ denotes the gradient. Evaluating both sides of this identity (which are 1forms) on the vector $\xi^{i}$, and using $v \xi^{i}=\lambda^{i} \xi^{i}$, one can see that the left hand side cancels with the second term on the right hand side, leading to $\xi^{i} \nabla \lambda^{i}=L_{\xi^{i}} \lambda^{i}=0$. This finishes the proof.

The requirement of the existence of characteristic integrals for all characteristic directions implies that all characteristic speeds must be linearly degenerate. Such systems are known as (totally) linearly degenerate, they have been thoroughly investigated in the literature, see e.g. $[14,24-26]$. For linearly degenerate systems the gradient catastrophe, which is typical for genuinely nonlinear systems, does not occur, and one has global existence results for an open set of initial data.

There exist systems which possess infinitely many characteristic integrals.

Example 2.1. The 2-component linearly degenerate system,

$$
v_{t}=w v_{x}, \quad w_{t}=v w_{x},
$$

possesses functionally many characteristic integrals in both characteristic directions:

$$
\frac{\phi(v)}{w-v}(d x+w d t), \quad \frac{\psi(w)}{v-w}(d x+v d t),
$$

here $\phi$ and $\psi$ are arbitrary functions of $v$ and $w$ respectively.

\section{Characteristic integrals of second order PDEs in 3D}

In this section we consider quasilinear wave-type equations of the form

$$
f_{11} u_{x x}+f_{22} u_{y y}+f_{33} u_{t t}+2 f_{12} u_{x y}+2 f_{13} u_{x t}+2 f_{23} u_{y t}=0,
$$

where $u(x, y, t)$ is a function of three independent variables, and the coefficients $f_{i j}$ depend on the first order derivatives $u_{x}, u_{y}, u_{t}$ only. Equations of this form generalise Examples 1.1 and 1.2 from 
the introduction. Their integrability by the method of hydrodynamic reductions [7] was investigated in [4]. We recall that, for broad classes of dispersionless PDEs in 3D including (3.1), the following conditions are equivalent:

- The equation is integrable by the method of hydrodynamic reductions $[7,11]$.

- The equation possesses a dispersionless Lax pair $[20,30]$ or, equivalently, a Lax pair in vector fields $[16,17]$.

- For every solution of the equation, the symbol of formal linearisation defines an EinsteinWeyl structure [10].

Either of these conditions leads to one and the same system of differential constraints for the coefficients $f_{i j}$, which was first derived in [4]. It was shown in [4] that any integrable equation of the form (3.1) possesses exactly four conservation laws

$$
\left(f_{i}\right)_{x}+\left(g_{i}\right)_{y}+\left(h_{i}\right)_{t}=0
$$

$i=1, \ldots, 4$, where $f_{i}, g_{i}, h_{i}$ are functions of $u_{x}, u_{y}, u_{t}$ only. Taking their linear combination with constant coefficients $J_{1}, \ldots, J_{4}$, and adding trivial conservation laws, we obtain the expression $\left(F_{1}\right)_{x}+\left(F_{2}\right)_{y}+\left(F_{3}\right)_{t}=0$ where

$$
F_{1}=J_{i} f_{i}-J_{5} u_{y}+J_{6} u_{t}+J_{8}, \quad F_{2}=J_{i} g_{i}+J_{5} u_{x}-J_{7} u_{t}+J_{9}, \quad F_{3}=J_{i} h_{i}-J_{6} u_{x}+J_{7} u_{y}+J_{10} .
$$

Although the constants $J_{5}-J_{10}$ give trivial contribution to conservation laws, they do effect nontrivially the characteristic condition, $\mathrm{Fg}^{-1} \mathrm{~F}^{t}=0$, where $g=f_{i j}$ is the $3 \times 3$ symmetric matrix of the corresponding principal symbol. The characteristic condition imposes a system of quadratic constraints for the coefficients $J_{1}, \ldots, J_{10}$ which, in linearly degenerate integrable cases, specify a Veronese threefold $V^{3} \subset \mathbb{P}^{9}$. For 3D equations of the form (3.1), the concept of linear degeneracy can be defined as follows. Looking for travelling wave solutions in the form $u(x, y, t)=u(\xi, \eta)+\zeta$ where $\xi, \eta, \zeta$ are arbitrary linear forms in the variables $x, y, t$, we obtain a second-order PDE for $u(\xi, \eta)$,

$$
a u_{\xi \xi}+2 b u_{\xi \eta}+c u_{\eta \eta}=0
$$

where the coefficients $a, b, c$ are certain functions of $u_{\xi}$ and $u_{\eta}$. Setting $v=u_{\xi}, w=u_{\eta}$, one can rewrite this PDE as a two-component system of hydrodynamic type. We say that Equation (3.1) is linearly degenerate if all its travelling wave reductions are linearly degenerate in the sense of Sect. 2. The condition of linear degeneracy is equivalent to the identity (set $u_{x}, u_{y}, u_{t}=p_{1}, p_{2}, p_{3}$ and consider $f_{i j}$ as functions of $\left.p_{1}, p_{2}, p_{3}\right)$ :

$$
f_{(i j, k)}=c_{(k} f_{i j)},
$$

here $f_{i j, k}=\partial_{p_{k}} f_{i j}, c_{k}$ is a covector, and brackets denote complete symmetrisation in $i, j, k$. Linearly degenerate integrable PDEs were classified in [9]: 
Theorem 3.1. The following examples constitute a complete list of linearly degenerate integrable PDEs of the form (3.1):

$$
\begin{gathered}
\mu u_{t} u_{x y}+v u_{y} u_{x t}+\eta u_{x} u_{y t}=0, \quad \mu+v+\eta=0, \\
u_{x x}+u_{x} u_{y t}-u_{y} u_{x t}=0 \\
u_{x y}+u_{y} u_{x t}-u_{x} u_{y t}=0 \\
u_{y y}+u_{x t}+u_{y} u_{t t}-u_{t} u_{y t}=0 \\
u_{x t}+u_{x} u_{y y}-u_{y} u_{x y}=0 \\
u_{t t}-u_{x x}-u_{y y}=0
\end{gathered}
$$

In different contexts, the canonical forms of Theorem 3.1 have appeared in $[1,5,16-22,29]$.

The main result of this section is the following.

\section{Theorem 3.2.}

(i) If a 3D quasilinear PDE of the form (3.1) possesses 'sufficiently many' characteristic integrals, then it must be linearly degenerate. Here 'sufficiently many' means that the corresponding vector $F$ satisfies no extra algebraic constraints other than the characteristic condition itself, $F g^{-1} F^{t}=0$.

(ii) Any linearly degenerate integrable PDE (3.1) possesses $V^{3}$-worth of characteristic integrals.

Proof. To demonstrate (i) we recall the result of [4] according to which the functions $F_{i}$ defining a conservation law must satisfy the identity $F_{(i, j)}=s f_{i j}$, where $F_{i, j}=\partial_{p_{j}} F_{i}$, brackets denote symmetrisation in $i, j$, and $s$ is a coefficient of proportionality (all entries are viewed as functions of $p$ 's). The characteristic constraint takes the form

$$
\left(f^{-1}\right)^{i j} F_{i} F_{j}=0,
$$

which can be rewritten as $f_{i j} F^{i} F^{j}=0$ where we use the notation $F_{i}=f_{i j} F^{j}$. Differentiating the characteristic condition by $p_{k}$ we obtain

$$
-\left(f^{-1}\right)^{i p} f_{p q, k}\left(f^{-1}\right)^{q j} F_{i} F_{j}+2\left(f^{-1}\right)^{i j} F_{i, k} F_{j}=0,
$$

which can be rewritten as

$$
f_{p q, k} F^{p} F^{q}=2 F_{i, k} F^{i} .
$$

Contracting this identity with $F^{k}$, using the condition $F_{(i, j)}=s f_{i j}$ and the characteristic constraint $f_{i j} F^{i} F^{j}=0$ we obtain the additional algebraic condition

$$
f_{i j, k} F^{i} F^{j} F^{k}=0 .
$$

The requirement that this condition is satisfied identically modulo the characteristic constraint, $f_{i j} F^{i} F^{j}=0$, is equivalent to saying that the cubic (3.3) is divisible by the quadric $f_{i j} F^{i} F^{j}=0$,

$$
f_{i j, k} F^{i} F^{j} F^{k}=\left(c_{i} F^{i}\right)\left(f_{i j} F^{i} F^{j}\right),
$$

for some linear form $c_{i} F^{i}$. Symmetrisation of this identity implies the condition of linear degeneracy (3.2). 
Finally, the proof of (ii) is a case-by-case calculation. Details are included in the Appendix. This finishes the proof of Theorem 3.2.

Note that linearly non-degenerate or non-integrable equations may also possess characteristic integrals, alhough not 'as many' as linearly degenerate integrable ones.

Example 3.1. The following integrable (linearly non-degenerate) equation,

$$
u_{t} u_{x y}+u_{y} u_{x t}+u_{x} u_{y t}=0
$$

admits only three characteristic conservation laws:

$$
\begin{aligned}
& \left(u_{x}^{2} u_{t}\right)_{y}+\left(u_{x}^{2} u_{y}\right)_{t}=0, \\
& \left(u_{y}^{2} u_{t}\right)_{x}+\left(u_{y}^{2} u_{x}\right)_{t}=0, \\
& \left(u_{t}^{2} u_{y}\right)_{x}+\left(u_{t}^{2} u_{x}\right)_{y}=0 .
\end{aligned}
$$

\section{Concluding remarks}

We have demonstrated that linearly degenerate second-order quasilinear integrable PDEs in 3D possess characteristic integrals parametrised by points on the Veronese variety $V^{3}$. Our calculations suggest that similar phenomena take place for other classes of linearly degenerate dispersionless integrable systems: all of them possess nontrivial characteristic integrals, what may change is the dimension of the corresponding Veronese variety.

Note that to be Darboux-integrable, the equation is required to possess sufficiently many higherorder characteristic integrals. The existence of such integrals in multi-dimensions is still an open problem.

\section{Appendix: characteristic integrals for equations from Theorem 3.1}

Here we present characteristic conservation laws for all examples from Theorem 3.1. For each of the canonical forms, we present four nontrivial conservation laws (which are found by direct calculation, recall that their existence follows from the general result of [4]), and parametric expressions for the corresponding constants $J_{1}, \ldots, J_{10}$ as defined in Sect. 3. In each case these parametric equations are readily seen to specify a Veronese threefold.

Equation 1 (discussed in the Introduction):

$$
\mu u_{t} u_{x y}+v u_{y} u_{x t}+\eta u_{x} u_{y t}=0 .
$$

Four conservation laws:

$$
\begin{gathered}
\eta\left(u_{y} u_{t}\right)_{1}+v\left(u_{x} u_{t}\right)_{y}+\mu\left(u_{x} u_{y}\right)_{t}=0 \\
v\left(\frac{u_{y}}{u_{t}}\right)_{1}+\eta\left(\frac{u_{x}}{u_{t}}\right)_{y}=0
\end{gathered}
$$


E.V. Ferapontov and J. Moss

$$
\begin{aligned}
& \mu\left(\frac{u_{t}}{u_{y}}\right)_{x}+\eta\left(\frac{u_{x}}{u_{y}}\right)_{t}=0, \\
& \mu\left(\frac{u_{y}}{u_{x}}\right)_{y}+v\left(\frac{u_{y}}{u_{x}}\right)_{t}=0 .
\end{aligned}
$$

Coefficients of characteristic integrals:

$$
\begin{gathered}
J_{1}=\alpha^{2}, \quad J_{2}=\frac{1}{4 v \eta} \beta^{2}, \quad J_{3}=\frac{1}{4 \eta \mu} \delta^{2}, \quad J_{4}=\frac{1}{4 v \mu} \gamma^{2}, \\
J_{5}=\alpha \beta, \quad J_{6}=\alpha \delta, \quad J_{7}=\alpha \gamma, \quad J_{8}=-\frac{1}{2 \eta} \beta \delta, \quad J_{9}=-\frac{1}{2 v} \beta \gamma, \quad J_{10}=-\frac{1}{2 \mu} \delta \gamma .
\end{gathered}
$$

\section{Equation 2.}

$$
u_{x x}+u_{x} u_{y t}-u_{y} u_{x t}=0 .
$$

Four conservation laws:

$$
\begin{gathered}
\left(\frac{u_{y}}{2 u_{x}^{2}}\right)_{x}+\left(\frac{1}{2 u_{x}}\right)_{y}-\left(\frac{u_{y}^{2}}{2 u_{x}^{2}}\right)_{t}=0, \\
\left(u_{x}-u_{y} u_{t}\right)_{x}+\left(u_{x} u_{t}\right)_{y}=0, \\
\left(2 u_{x} u_{t}-u_{y} u_{t}^{2}\right)_{x}+\left(u_{t}^{2} u_{x}\right)_{y}-\left(u_{x}^{2}\right)_{t}=0, \\
-\left(\frac{1}{u_{x}}\right)_{x}+\left(\frac{u_{y}}{u_{x}}\right)_{t}=0 .
\end{gathered}
$$

Coefficients of characteristic integrals:

$$
\begin{gathered}
J_{1}=\alpha^{2}, \quad J_{2}=-\delta \beta, \quad J_{3}=\frac{1}{2} \beta^{2}, \quad J_{4}=\alpha \gamma \\
J_{5}=\frac{1}{2} \delta^{2}, \quad J_{6}=\beta \gamma, \quad J_{7}=\alpha \beta, \quad J_{8}=-\alpha \beta-\gamma \delta, \quad J_{9}=\alpha \delta, \quad J_{10}=-\frac{1}{2} \gamma^{2} .
\end{gathered}
$$

\section{Equation 3.}

$$
u_{x y}+u_{y} u_{x t}-u_{x} u_{y t}=0 .
$$

Four conservation laws:

$$
\begin{gathered}
\left(u_{y} u_{t}\right)_{x}+\left(u_{x}-u_{x} u_{t}\right)_{y}=0 \\
\left(u_{y} u_{t}^{2}\right)_{x}+\left(2 u_{x} u_{t}-u_{x} u_{t}^{2}-u_{x}\right)_{y}-\left(u_{x} u_{y}\right)_{t}=0 \\
\text { Co-published by Atlantis Press and Taylor \& Francis } \\
\text { Copyright: the authors }
\end{gathered}
$$




$$
\begin{aligned}
& \left(\frac{1}{u_{y}}\right)_{x}-\left(\frac{u_{x}}{u_{y}}\right)_{t}=0, \\
& -\left(\frac{1}{u_{x}}\right)_{y}-\left(\frac{u_{y}}{u_{x}}\right)_{t}=0 .
\end{aligned}
$$

Coefficients of characteristic integrals:

$$
\begin{gathered}
J_{1}=\alpha \beta, \quad J_{2}=\alpha^{2}, \quad J_{3}=\frac{1}{4} \delta^{2}, \quad J_{4}=\frac{1}{4} \gamma^{2} \\
J_{5}=-\frac{1}{4} \beta^{2}, \quad J_{6}=-\alpha \delta, \quad J_{7}=\alpha \gamma, \quad J_{8}=-\frac{1}{2} \beta \delta, \quad J_{9}=\alpha \gamma-\frac{1}{2} \beta \gamma, \quad J_{10}=-\frac{1}{2} \delta \gamma .
\end{gathered}
$$

\section{Equation 4.}

$$
u_{y y}+u_{x t}+u_{y} u_{t t}-u_{t} u_{y t}=0 .
$$

Four conservation laws:

$$
\begin{gathered}
\left(u_{y}-u_{t}^{2}\right)_{y}+\left(u_{x}+u_{y} u_{t}\right)_{t}=0, \\
\left(\frac{1}{2} u_{t}^{2}\right)_{x}+\left(u_{y} u_{t}-\frac{1}{2} u_{t}^{3}\right)_{y}+\left(-u_{y} u_{t}^{2}-\frac{1}{2} u_{y}^{2}+\frac{3}{2} u_{t}^{2} u_{y}\right)_{t}=0, \\
\left(u_{y} u_{t}-u_{t}^{3}\right)_{x}+\left(-u_{x} u_{t}+u_{y}^{2}-3 u_{y} u_{t}^{2}+u_{t}^{4}\right)_{y}+\left(u_{x} u_{y}+2 u_{t} u_{y}^{2}-u_{y} u_{t}^{3}\right)_{t}=0, \\
\left(u_{y}^{2}-2 u_{y} u_{t}^{2}+u_{t}^{4}\right)_{x}+\left(-2 u_{x} u_{y}+2 u_{x} u_{t}^{2}-3 u_{y}^{2} u_{t}+4 u_{y} u_{t}^{3}-u_{t}^{5}\right)_{y}+\left(-2 u_{x} u_{y} u_{t}-u_{x}^{2}-3 u_{y}^{2} u_{t}^{2}+u_{y} u_{t}^{4}+u_{y}^{3}\right)_{t}=0 .
\end{gathered}
$$

Coefficients of characteristic integrals:

$$
\begin{gathered}
J_{1}=-\alpha \delta-\frac{1}{2} \beta \gamma, \quad J_{2}=2 \alpha \gamma+\frac{1}{2} \delta^{2}, \quad J_{3}=\frac{1}{2} \delta \gamma, \quad J_{4}=\frac{1}{4} \gamma^{2}, \\
J_{5}=\alpha \gamma, \quad J_{6}=-\alpha \delta, \quad J_{7}=\alpha^{2}+\frac{1}{2} \beta \delta, \quad J_{8}=\alpha^{2} \quad J_{9}=\alpha \beta, \quad J_{10}=-\frac{1}{4} \beta^{2} .
\end{gathered}
$$

\section{Equation 5.}

$$
u_{x t}+u_{x} u_{y y}-u_{y} u_{x y}=0 .
$$

Four conservation laws:

$$
\begin{gathered}
\left(\frac{u_{y}}{u_{x}}\right)_{y}-\left(\frac{1}{u_{x}}\right)_{t}=0, \\
\left(-u_{y}^{2}\right)_{1}+\left(u_{x} u_{y}\right)_{y}+\left(u_{x}\right)_{t}=0, \\
\left(-u_{t}^{2}+2 u_{t} u_{y}^{2}-u_{y}^{4}\right)_{x}+\left(-2 u_{x} u_{y} u_{t}+u_{x} u_{y}^{3}\right)_{y}+\left(u_{x} u_{y}^{2}\right)_{t}=0, \\
\text { Co-published by Atlantis Press and Taylor \& Francis } \\
\text { Copyright: the authors }
\end{gathered}
$$




$$
\left(u_{t} u_{y}-u_{y}^{3}\right)_{x}+\left(-u_{x} u_{t}+u_{x} u_{y}^{2}\right)_{y}+\left(u_{x} u_{y}\right)_{t}=0 .
$$

Coefficients of characteristic integrals:

$$
\begin{gathered}
J_{1}=\alpha^{2}, \quad J_{2}=-\frac{1}{2} \beta \gamma-\frac{1}{4} \delta^{2}, \quad J_{3}=-\frac{1}{4} \beta^{2}, \quad J_{4}=-\frac{1}{2} \beta \delta, \\
J_{5}=-\frac{1}{2} \gamma \delta, \quad J_{6}=-\frac{1}{2} \beta \gamma, \quad J_{7}=\beta \alpha, \quad J_{8}=\frac{1}{4} \gamma^{2}, \quad J_{9}=\gamma \alpha, \quad J_{10}=\delta \alpha .
\end{gathered}
$$

\section{References}

[1] V.E. Adler and A.B. Shabat, Model equation of the theory of solitons, Theoret. and Math. Phys. 153 (2007) 1373-1387.

[2] C. Athorne, A $Z^{2} \times R^{3}$ Toda system, Phys. Lett. A 206 (1995) 162-166.

[3] C. Athorne and H. Yilmaz, Laplace invariants for general hyperbolic systems, J. Nonlinear Math. Phys. 19 (2012) 1250024.

[4] P.A. Burovskii, E.V. Ferapontov and S.P. Tsarev, Second order quasilinear PDEs and conformal structures in projective space, International J. Math. 21 (2010) 799-841.

[5] M. Dunajski, A class of Einstein-Weyl spaces associated to an integrable system of hydrodynamic type. J. Geom. Phys. 51 (2004) 126-137.

[6] M. Dunajski and W. Krynski, Einstein-Weyl geometry, dispersionless Hirota equation and Veronese webs, arXiv:1301.0621.

[7] E.V. Ferapontov and K.R. Khusnutdinova, On integrability of (2+1)-dimensional quasilinear systems, Comm. Math. Phys. 248 (2004) 187-206.

[8] E.V. Ferapontov, K.R. Khusnutdinova and S.P. Tsarev, On a class of three-dimensional integrable Lagrangians, Comm. Math. Phys. 261 (2006) 225-243.

[9] E.V. Ferapontov and J. Moss, Linearly degenerate PDEs and quadratic line complexes, arXiv:1204.2777.

[10] E.V. Ferapontov and B. Kruglikov, Dispersionless integrable systems in 3D and Einstein-Weyl geometry, to appear in Journal of Differential Geometry (2014); arXiv:1208.2728v1.

[11] J. Gibbons and S.P. Tsarev, Reductions of the Benney equations, Phys. Lett. A 211 (1996) 19-24.

[12] D. Hartley, Darboux integrability in more than two dimensions, AAECC 11 (2001) 397-416.

[13] K. Kakié, On involutive systems of partial differential equations whose characters of order more than one vanish, Proc. Japan Acad. 51 (1975) 265-269.

[14] T.P. Liu, Development of singularities in the nonlinear waves for quasi-linear hyperbolic PDEs, J. Diff. Eq. 33 (1979) 92-111.

[15] A. Majda, Compressible fluid flows and systems of conservation laws in several space variables, Appl. Math. Sci. 53 (Springer, New York, 1984).

[16] S.V. Manakov and P.M. Santini, Inverse scattering problem for vector fields and the Cauchy problem for the heavenly equation, Phys. Lett. A 359 (2006) 613-619.

[17] S.V. Manakov and P.M. Santini, On the solutions of the second heavenly and Pavlov equations, J. Phys. A 42 (2009) 404013.

[18] L. Martinez Alonso and A.B. Shabat, Hydrodynamic reductions and solutions of a universal hierarchy, Theoret. and Mat. Phys. 140 (2004) 1073-1085.

[19] O.I. Morozov, Recursion Operators and Nonlocal Symmetries for Integrable rmdKP and rdDym Equations, arXiv:1202.2308.

[20] A. Odesskii and V. Sokolov, Integrable $(2+1)$-dimensional systems of hydrodynamic type, Theoretical and Mathematical Physics 163 (2010) 549-586.

[21] V. Ovsienko, Bi-Hamiltonian nature of the equation $u_{t x}=u_{x y} u_{y}-u_{y y} u_{x}$, Adv. Pure Appl. Math. 1 (2010) $7-17$. 
[22] M.V. Pavlov, Integrable hydrodynamic chains, J. Math. Phys. 44 (2003) 4134-4156.

[23] D.H. Parsons, The extension of Darboux's method, Mémor. Sci. Math., Fasc. 142 (Gauthier-Villars, Paris, 1960).

[24] B.L. Rozdestvenskii and A.D. Sidorenko, On the impossibility of 'gradient catastrophe' for weakly nonlinear systems, Z. Vycisl. Mat. i Mat. Fiz. 7 (1967) 1176-1179.

[25] B.L. Rozdestvenskii and N.N. Yanenko, Systems of quasilinear equations and their applications to gas dynamics, translated from the second Russian edition by J. R. Schulenberger, Translations of Mathematical Monographs 55 (American Mathematical Society, Providence, RI, 1983).

[26] D. Serre, Systems of conservation laws. 1. Hyperbolicity, entropies, shock waves (Cambridge University Press, 1999); Systems of conservation laws. 2. Geometric structures, oscillations, and initial-boundary value problems (Cambridge University Press, 2000).

[27] V.V. Sokolov and A.V. Zhiber, On the Darboux integrable hyperbolic equations, Phys. Lett. A 208 (1995) 303-308.

[28] S.P. Tsarev, Generalized Laplace transformations and integration of hyperbolic systems of linear partial differential equations, in Proc. Int. Symp. Symbolic and Algebraic Computation, ISSAC 2005 (Beijing, China, July 24-27, 2005) (ACM Press, 2008), pp. 325-331.

[29] I. Zakharevich, Nonlinear wave equation, nonlinear Riemann problem, and the twistor transform of Veronese webs, arXiv:math-ph/0006001.

[30] V.E. Zakharov, Dispersionless limit of integrable systems in $2+1$ dimensions, in Singular Limits of Dispersive Waves, Ed. N.M. Ercolani et al. (Plenum Press, New York, 1994).

[31] A.V. Zhiber and V.V. Sokolov, Exactly integrable hyperbolic equations of Liouville type, Uspekhi Mat. Nauk 56 (2001) 63-106. 